\title{
Tres Versiones de DaMa DETRÁs de UN PARAPETO: INVESTIGACIÓN MULTIDISCIPLINAR DE LA MENOS CONOCIDA Y NUEVA PROPUESTA DE ATRIBUCIÓN
}

\section{"Three versions of Lady behind a parapet: \\ multidisciplinary research of the one least known and new attribution proposal"}

\section{Concepción Chillón Domínguez, S. Ruiz-Moreno ${ }^{1}$}

Recibido: 07-05-2020

Aceptado: 20-07-2020

\section{Resumen}

En este trabajo se presenta la investigación de un retrato femenino renacentista que ha estado ilocalizable durante muchos años y, en consecuencia, inaccesible a los investigadores. Se trata de una tercera versión del tema Dama detrás de un parapeto, título con el que se conocen también las obras hoy en día atribuidas a Giovanni Cariani y conservadas en la Galería Estense de Módena y en el Museo de Bellas Artes de Budapest. Los resultados obtenidos en la investigación de esta tercera versión, actualmente en Barcelona, han aportado datos inéditos en cuanto a su historia, realidad físico-química, iconografía y estilo que permiten proponer una nueva hipótesis en cuanto a su atribución.

Palabras clave: Renacimiento, pintura veneciana, retrato, análisis científicos.

\begin{abstract}
In this paper, the investigation of a Renaissance feminine portrait is presented. Due to its unknown location for many decades, this oil painting has been inaccessible for a long time for the academic community. It deals with a third version of the topic Lady behind a parapet, title with which the two versions located at Estense Gallery of Modena and at Museum of Fine Arts of Budapest are traditionally known and both attributed to Giovanni Cariani. The set of scientific and documental results obtained in the research of this third version (today in Barcelona) provides unpublished information as for its history, basic pigmentation, iconography and style, which allow us to propose a new hypothesis about its attribution.
\end{abstract}

Keywords: Renaissance, venetian painting, portrait, scientific analysis.

1 Departamento de Teoría de la Señal y Comunicaciones (TSC), Universitat Politècnica de Catalunya. *conchillon@hotmail. com; sruiz@tsc. upc. edu. Los autores agradecen especialmente las aclaraciones y aportaciones de Matteo Mancini (Departamento de Historia del Arte de la Universidad Complutense, Madrid), de Victoria Reed (Museum of Fine Arts, Boston) y de Nikoletta Koruhely (Museum of Fine Arts, Budapest), así como al Grupo de Comunicaciones Ópticas de la U. P. C. por su ayuda en las tareas experimentales. 


\section{Introducción}

"La pintura contiene una fuerza divina, pues logra que estén presentes los ausentes"2

En la primera mitad del siglo XVI se pintaron en Venecia tres versiones muy parecidas de un retrato femenino conocido como Dama detrás de un parapeto. Una de ellas se conserva en el Museo de Bellas Artes de Budapest (Fig. 1), otra en la Galería Estense de Módena (Fig. 2) y la tercera pertenece a una colección particular de Barcelona (Fig. 3). Esta última versión estuvo en paradero desconocido durante muchos años hasta que los actuales propietarios propusieron a la Universidad Politécnica de Cataluña realizar una investigación multidisciplinar que comenzó, en primer lugar, con el análisis de los elementos iconográficos más relevantes de la obra, como el parapeto y la letra "V" inscrita en él, poniendo de manifiesto también las diferencias y similitudes entre las tres versiones. A continuación, ya en laboratorio, se analizó la superficie de la pintura tanto con espectroscopia Raman como con reflectometría infrarroja. ${ }^{3}$ La primera técnica se aplicó con el fin de identificar de manera no destructiva los pigmentos de la obra y la segunda, para obtener información acerca de los trazos realizados al carbón, tanto subyacentes como superficiales. ${ }^{4}$ Finalmente, se investigaron, en la medida que fue

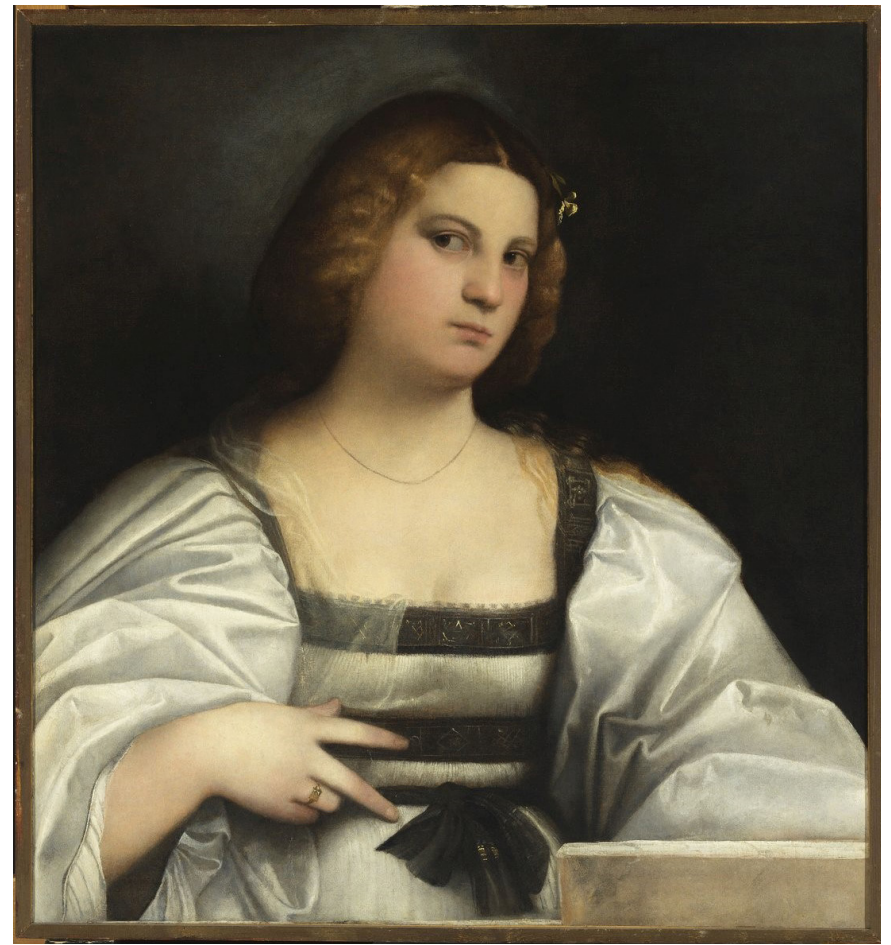

Fig. I: Dama detrás de un parapeto. Óleo y temple sobre lienzo, $64.5 \mathrm{~cm}$. x $60 \mathrm{~cm}$. Museo de Bellas Artes, Budapest.

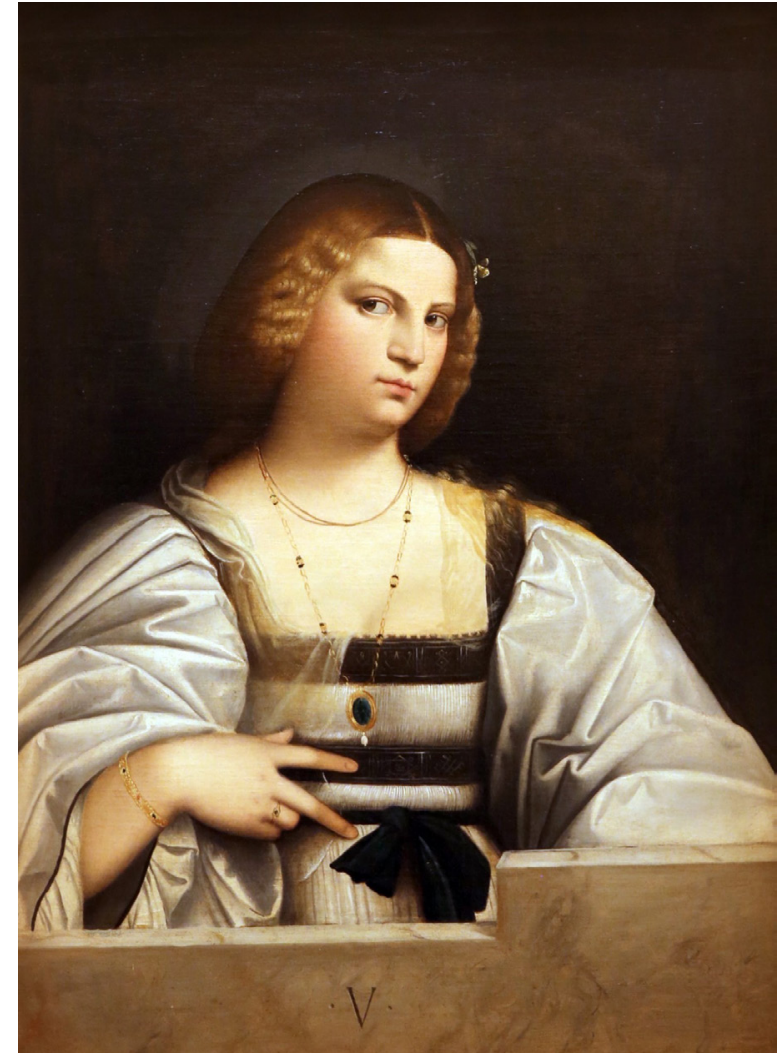

Fig. 2: Dama detrás de un parapeto. Óleo sobre lienzo, $89 \mathrm{~cm} . \times 65 \mathrm{~cm}$. Galería Estense, Módena.

2 ALBERTI, L. B. Tratado de pintura. México D. F. : Universidad Autónoma Metropolitana, 1988, p. 77

3 Para conocer los espectros de los pigmentos más utilizados en pintura antes de 1850, ver: BELL, I. M. , CLARK, R. J. H. y GIBBS, P. J. Raman spectroscopy library of natural and synthetic pigments (pre-1850). Spectrochimica Acta. Part A, 1997, 53, pp. $2159-2179$.

4 CHILLÓN, C. , FERRER, P. , RUIZ. MORENO, S. y LÓPEZ GIL, A. Raman spectroscopy: a fundamental tool in the analysis of artworks. En: R. AMOÊDA, S. LIRA y C. PINHEIRO, eds. Heritage 2012, 3rd International Conference on Heritage and Sustainable Development, vol. 2. Portugal: Green Lines Institute, 2012, pp. 805-814. 
posible, la historia y procedencia de la obra, así como la controversia sobre las diferentes atribuciones de autoría que realizaron diversos historiadores. Con todo ello, se han conseguido aportar datos científicos y documentales inéditos que demuestran que la versión de Barcelona es, además de la de mayor calidad, la que se realizó en primer lugar y, consecuentemente, debió servir de modelo a las versiones de Módena y Budapest.

\section{El retrato con parapeto en el Renacimiento}

Con la eclosión del Renacimiento, el retrato, como género artístico, fue ocupando un lugar muy destacado en el arte de la pintura. La cita de Alberti al inicio de este trabajo es un ejemplo de cómo el retrato tenía un carácter memorial o recordato-

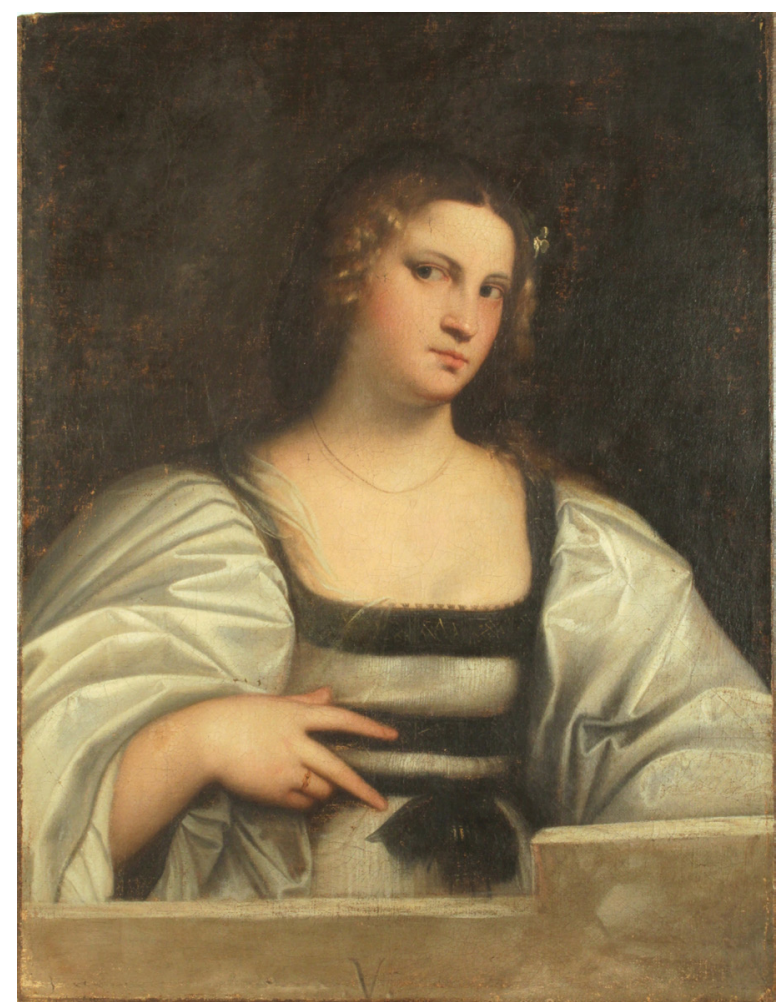

Fig. 3: Dama detrás de un parapeto. Óleo sobre lienzo, $74.5 \mathrm{~cm}$ x $59 \mathrm{~cm}$. Colección particular, Barcelona. rio, al igual que en la Roma clásica, donde la imagen de los antepasados era muy importante. Poco a poco, los retratos se fueron enriqueciendo (a menudo por requerimiento del propio comitente) con elementos iconográficos concretos que aportaban un significado simbólico. Uno de estos elementos iconográficos era un muro o parapeto tras el cual aparecía el personaje retratado. Considerando la influencia del humanismo y la recuperación del mundo clásico, es normal que ese muro fuera un recurso habitual en el Renacimiento ya que se trata de una derivación del modelo de los monumentos funerarios de la antigua Roma cuya finalidad era mantener al difunto vivo en el recuerdo. ${ }^{5}$ Esa intención de inmortalizar fue quizás lo que potenció también que el retrato ganara en importancia, no con el trasfondo funerario que tenía en Roma, pero sí con el objetivo de perpetuar la imagen del modelo, enaltecer valores y expresar conceptos universales. ${ }^{6}$ Algunos de los artistas más representativos del siglo XVI utilizaron el recurso del parapeto en sus retratos, como, por ejemplo, el gran Giorgio Barbarelli (Giorgione) en Retrato Giustiniani (c. 1505) ${ }^{7}$ y Retrato de hombre joven (c. 1508-1510), ${ }^{8}$ Tiziano en los retratos de Ariosto (1510) y La Schiavona (1510-12), ${ }^{9}$ Palma el Viejo en La Bella

5 THOMPSON, N. VV and related inscriptions in Giorgione, Titian and Dürer. The Art Bulletin, 1975, 57, p. 349 [consulta: 7 de junio de 2020]. Disponible en: https://www.jstor.org/stable/pdf/3049402.pdf?refreqid=excelsior\%3A25a22fb7404e06a969ec59c9630b0ad2. 6 SYSON, L. Testimonio de rostros, recuerdo de almas. En: FALOMIR, M. ed. El retrato del Renacimiento. Madrid: El Viso, 2008, pp. 23-39.

7 En la Gemäldegalerie de Berlín. Óleo sobre lienzo, $58 \mathrm{~cm}$. x $46 \mathrm{~cm}$. N INV. 12 A.

8 En el Museo de Bellas Artes de Budapest. También conocido como Retrato de Antonio Broccardo. Óleo sobre lienzo, $72.5 \mathrm{~cm}$. x 54 cm. No INV. 94.

9 Ambos en la National Gallery de Londres. Ariosto, también conocido como Retrato de Gerolamo Barbarigo es un óleo sobre lienzo de $81.2 \mathrm{~cm}$. x 66. $3 \mathrm{~cm}$. N ${ }^{\circ}$ INV. NG1944; La Schiavona, también óleo sobre lienzo, es de mayores dimensiones, $119.4 \mathrm{~cm}$. x 96.5 cm. N ${ }^{o}$ INV. NG5385. 
(c. 1518-1520) ${ }^{10}$ y Giovanni Cariani en el Retrato de hombre veneciano (c. 1510-1515) ${ }^{11}$ y en el Retrato de hombre joven (c. 1515). ${ }^{12}$ A este conjunto de retratos, que destacan por la particular tipología de añadir un muro con letras inscritas en primer término, se deben añadir las tres versiones del retrato que se han presentado anteriormente, la menos conocida de las cuales ha sido el motivo fundamental de la presente investigación.

\section{Dama detrás de un parapeto: estudio artístico y análisis científico}

La obra investigada muestra a una joven sobre fondo neutro y ubicada detrás de un parapeto en el que se aprecia inscrita la letra "V". La dama, de rostro bello y delicado, tiene el cabello rubio y lo lleva suelto y adornado con una sencilla flor de azahar, símbolo de castidad y pureza. Su vestido pertenece a la moda típicamente veneciana de principios del Cinquecento, ${ }^{13}$ con anchas y voluminosas mangas, ${ }^{14}$ escote recto y bordado en oro sobre franja negra, velo transparente sobre el pecho y un lazo negro ciñéndole la cintura. La joven lleva la mirada hacia su lado derecho mientras señala hacia su vientre con los dedos índice y corazón formando una "V", aspecto que también puede estar relacionado con su carácter puro y virginal. Su estatus de dama se muestra en un pequeño anillo que luce en el dedo anular de su mano derecha.

El lienzo, aunque se encuentra en un excelente estado de conservación, presenta pequeñas lagunas pictóricas que, con ayuda de una binocular, permiten visualizar una capa de imprimación de tono amarronado o rojizo, práctica habitual en los siglos XVI y XVII. ${ }^{15}$

En la parte posterior de la obra se pueden distinguir dos sellos, aunque el paso del tiempo ha hecho que actualmente sean difícilmente legibles. En uno de ellos se ha podido leer "USA PATD. 2, 491, 600 Charles G. Bearce, 87 Porter ST. Melrose, Mass.", que corresponde a una patente registrada en Massachusetts en diciembre de 1949. ${ }^{16}$ El responsable de esta patente, Charles Gordon Bearce, inventó un tipo de bastidor (que incorpora unos extensores especiales) en el que el lienzo se encuentra montado actualmente. El otro sello, y según opinión del historiador del arte Matteo Mancini, del que corresponde al Ministero della pubblica istruzione esportazioni oggetti di antichitae darti cuya ley

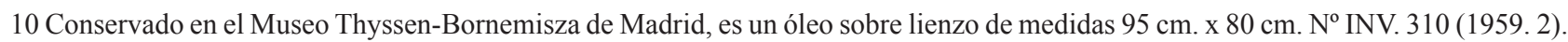

11 En la National Gallery of Art de Washington. Óleo sobre lienzo, 76. 2 cm. x 63.5 cm. № INV 1939. 1. 258. También atribuido a Giorgione y a la primera época de Tiziano.

12 En la Pinacoteca de Brera de Milán. Óleo sobre lienzo, 71 cm. x 57 cm. No INV. 2223.

13 MARANGON, G. Las cortesanas venecianas y la moda. En: Mujer, cultura y comunicación: realidades e imaginarios. IX Simposio Internacional de la Asociación Andaluza de Semiótica. Sevilla: Alfar, 2001, pp. 518-522 [consulta: 8 de junio de 2020]. ISBN 8489673-84-5. Disponible en: https://idus.us.es/bitstream/handle/11441/60783/MARANGON_Giorgia.pdf?sequence=1\&isAllowed=y.

14 MÁRQUEZ, J. La moda femenina en Italia del siglo XVI al siglo XVIII. En: La moda femenina en la Edad Moderna. Universidad de Cádiz, 2013 [consulta: 8 de junio de 2020]. Disponible en: https://www.academia.edu/5111954/LA_MODA_FEMENINA_EN LA EDAD MODERNA.

15 GAYO, M. D. y JOVER, M. Evolución de las preparaciones en la pintura de los siglos XVI y XVII en España. Boletín del Museo del Prado, 2010, 28 (46), pp. 39-59.

16 BEARCE, Ch. G. Frame for artists' canvases with expanders. US2491600A, United States Office, 1949 [consulta: 9 de junio de 2020]. Disponible en: https://patents.google.com/patent/US2491600A/en. 
entró en vigor el año 1939. ${ }^{17}$ Este sello indica que, a partir de esa fecha, la obra salió legalmente de Italia bajo un procedimiento oficial de exportación de patrimonio.

Por otra parte, y como ya se ha comentado, uno de los elementos iconográficos más característicos de esta obra, además del parapeto, es la letra "V" que aparece inscrita en su parte inferior. Un artículo de Nancy Thompson afrontó precisamente la presencia de la "V" en varias obras ejecutadas en Venecia durante la primera década del siglo XVI, las cuales ya han sido citadas anteriormente. ${ }^{18}$ Dos de ellas son Retrato de Ariosto y La Schiavona, en cuyos parapetos se pueden leer las letras "TV" que, en este caso, representan las iniciales de Tiziano Vecellio. Thompson también destacó el Retrato Giustiniani de Giorgione, en el que aparecen las letras "VV", el Retrato de hombre veneciano, actualmente atribuido a Giovanni Cariani, ${ }^{19}$ con las letras "VVO" y el Retrato de hombre joven, también de Giorgione, con la letra "V".

Thompson relacionó la letra "V" de las obras citadas con el adjetivo latino "vivus" (viviente) asociado a los monumentos funerarios de la Roma clásica que representaban el símbolo de la inmortalidad del difunto. No obstante, y sin descartar esa interpretación, parece más plausible la hipótesis que sugirió Maurizio Calvesi en 1970. ${ }^{20}$ Según él, las letras "V", "VV" y "VVO", presentes en los retratos que se han citado, aluden a la Verdad y a la Virtud, conceptos relacionados con el lema latino Virtus Ommnia Vincit, ampliamente utilizado en círculos humanísticos durante los siglos XVI y XVII, que significa "La Virtud todo lo vence". Así pues, la "V" inscrita en el muro de la obra investigada correspondería a la Virtud de la retratada, aspecto que es coherente con el resto de símbolos que aparecen en el retrato (como la flor de azahar) y que permite establecer que la obra representa una alegoría de la Virtud o bien un retrato matrimonial. Esta interpretación queda reforzada por la posición de los dedos en forma de "V" invertida que la dama dirige hacia su vientre, un gesto que Mauro Zanchi ha asociado a la virginidad y que está vinculado con el culto a la diosa Diana, símbolo de la castidad y personificación de la Luna. ${ }^{21}$ A pesar de que ese gesto no aparece recogido en el famoso manual sobre el significado de posiciones de dedos y manos que Giovanni Bonifacio compiló en el siglo XVII, ${ }^{22}$ efectivamente existen suficientes ejemplos en obras del Cinquecento y del Seicento (por ejemplo, el Retrato de joven como virgen sabia (c. 1510) de Sebastiano del Piombo y la Diana cazadora (1658) de Guercino) que demuestran, según la tesis de Zanchi, que pudo existir un cierto culto de origen pagano a la diosa Diana, aspecto que reafirma que la obra investigada, más allá de la mera representación retratística, evoca los conceptos de Virtud y Virginidad.

17 Legge 1 Giugno 1939, $\mathrm{n}^{\circ} .1089$. Tutela della cose d'interesse artistico o storico, en: Gazzeta Uffiziale della Repubblica italiana, $\mathrm{n}^{\circ}$ 184, 8 de agosto de 1939 [consulta: 9 de junio de 2020]. Disponible en: https://librari.beniculturali.it/export/sites/dgbid/it/documenti/ Normativa/Legge 1 giugno 1939 n 1089.pdf.

18 THOMPSON, ref. 5, pp. 346-356.

19 Esta obra también ha estado atribuida a Giorgione y a Tiziano (incluso a ambos). Actualmente ver: https://nga.gov/collection/art-object-page.399.html [consulta: 9 de junio de 2020].

20 CALVESI, M. La morte di bacio. Saggio sull'ermetismo di Giorgione. Storia dell'Arte, 1970, 7-8, pp. 179-233.

21 ZANCHI, M. Sotto il segno di Diana. Il gesto a V rovesciata nell'arte del Cinquecento: letture iconologiche. Art e dossier, 2014, $\mathrm{n}^{\circ} 315$, pp. 72-77.

22 BONIFACIO, G. L'Arte de Cenni. Vicenza, 1616 [consulta: 9 de junio de 2020]. Disponible en: https://archive.org/details/lartedecenniconl02boni/page/n3/mode/2up. 
Por otra parte, a pesar de las similitudes existentes entre las tres versiones, son apreciables también algunas diferencias de ejecución y de estilo. Por ejemplo, en la versión de Módena (Fig. 2) la retratada luce un brazalete y un colgante, joyas que no aparecen ni en la versión de Budapest ni en la de Barcelona. En la versión de Budapest (Fig. 1) no existe la "V" porque, según información aportada por Nikoletta Koruhely, conservadora del Departamento de Grandes Maestros del Museo de Bellas Artes de Budapest, el lienzo fue recortado en fecha desconocida, un aspecto que se puso de manifiesto en el trabajo de restauración que realizó Nicolás More y que se publicó en 1979. ${ }^{23}$ More, entre otros aspectos, destacó que la versión de Budapest había sido objeto de una restauración anterior, en 1966, y que tenía una fina capa de preparación de tono amarronado, elemento común con la versión de Barcelona. En cuanto al estilo, More opinaba que, tanto la versión de Budapest como la conservada en Módena, debían ser copias de un modelo original, si bien la obra de Budapest debió ser más fiel o exacta pues la versión de Módena presenta una factura más rígida y menos natural.

Comparando las tres versiones, en las de Budapest y Módena la retratada presenta un rostro más ancho y un semblante más serio y menos inocente que en la versión de Barcelona. En esta última destaca una expresión dulce e ingenua, más acorde con el simbolismo iconográfico que representa y, en general, es notable una mayor calidad respecto a las otras dos en cuanto al tratamiento de luces y sombras, a la naturalidad en el volumen del ropaje y en el cuidado de los rasgos faciales. Una sencilla comparación visual entre los tres retratos permite apreciar que las versiones de Budapest y Módena son estilísticamente más parecidas entre sí y denotan menor calidad que la versión investigada.

La calidad artística de la versión de Barcelona también se constató realizando un exhaustivo análisis con reflectometría infrarroja (I. R.) gracias al cual se pudo apreciar un sutil dibujo preliminar realizado al carbón en la silueta de la cabeza (Fig. 4), de la mano y de cada dedo, así como del perfil del rostro, de los pliegues del vestido y de las líneas del parapeto.

La investigación científica se completó con un análisis no destructivo de la pigmentación con espectroscopia Raman. Para ello se analizaron directamente (esto es, sin extracción alguna de muestras)

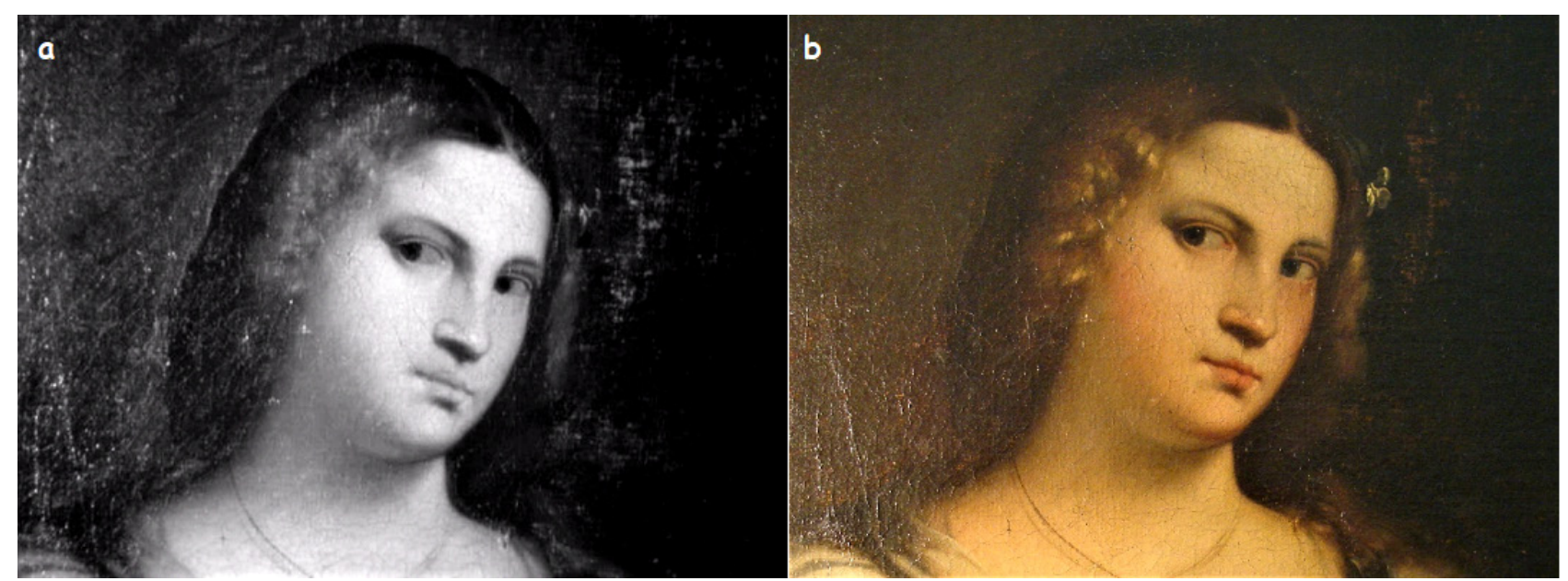

Fig. 4: Dama detrás de un parapeto. Fotografía IR (a) comparada con su imagen visible (b). Colección particular, Barcelona.

23 MORE, N. Résumé de la restauration du tableau "Violante" de Giovanni Cariani. Bulletin du Musée Hongrois des Beaux-Arts, 1979, 52, pp. 89-96 [consulta: 9 de junio de 2020]. Disponible en: https://library.hungaricana.hu/en/view/ORSZ_SZEP_Kozl_053/?query=More $\% 2 \mathrm{C} \% 20$ Nicolas\&pg $=90 \&$ layout $=\mathrm{S}$. 


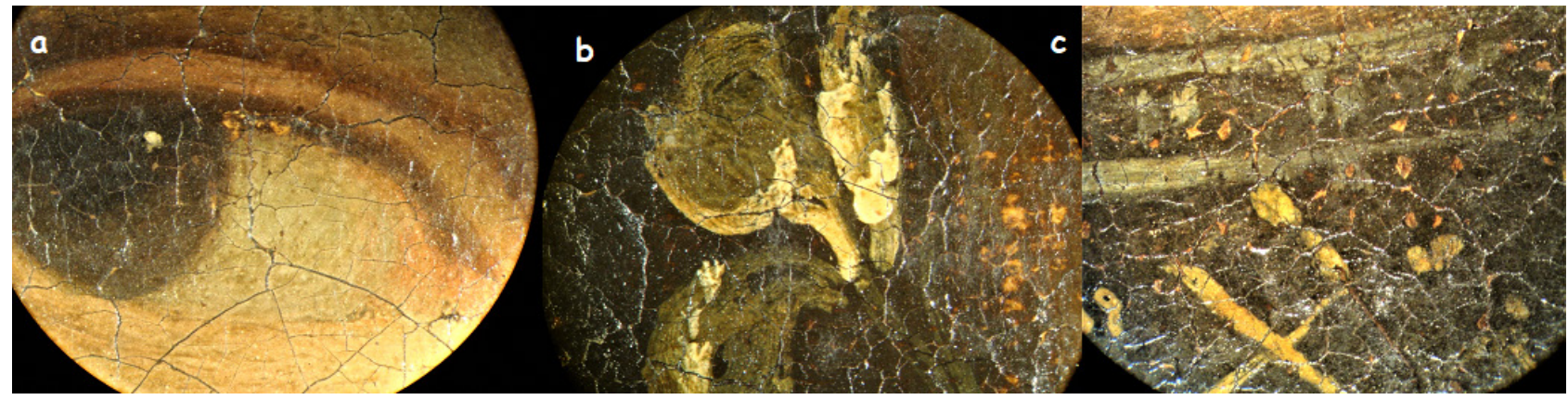

Fig. 5: Dama detrás de un parapeto. Detalles de: ojo derecho (a), flor de azahar (b) y decoración del vestido (c). Colección particular, Barcelona.

varias zonas cromáticamente representativas de la obra y se identificaron los siguientes pigmentos: blanco de plomo en el vestido, flor de azahar, ojos y carnaciones; óxido férrico en carnaciones, labios y lagrimales; hidróxido férrico en el pelo, anillo y bordados del vestido; y carbón en el cinturón y pectoral del vestido. Estos pigmentos fueron identificados tanto aislados como mezclados entre sí.

El blanco de plomo, carbonato básico de plomo, $2 \mathrm{PbCO} 3 \mathrm{~Pb}(\mathrm{OH}) 2$, también conocido como albayalde, fue sintetizado ya en la antigüedad y es uno de los pigmentos sintéticos e históricos de mayores cualidades artísticas, siendo el principal pigmento blanco utilizado en pintura al óleo hasta la aparición del blanco de cinc en la segunda mitad del XIX. ${ }^{24}$ Las tierras férricas identificadas han sido hematites (óxidos férricos) y ocres (hidróxidos férricos), ambas muy habituales en pintura al óleo y utilizadas desde la antigüedad (sus versiones sintéticas aparecieron en el siglo XIX) ${ }^{25}$ Finalmente, el negro de carbón, tanto de origen animal como vegetal, se usa como pigmento en Europa desde la época de las pinturas rupestres $\mathrm{y}$, debido a su inmejorable calidad, nunca ha dejado de utilizarse ni ha sido superado por otros pigmentos negros. ${ }^{26}$

Aunque esta paleta puede parecer austera y limitada, en realidad demuestra la capacidad técnica del artista para realizar mezclas y crear una gran variedad de tonalidades (Fig. 5). Por otra parte, por el uso habitual y constante en el tiempo de estos pigmentos (también conocidos como pigmentos históricos), se trata de una paleta perfectamente coherente con la primera década del siglo XVI, época en la que, según estilo y tipología, se ha fechado la realización de este retrato. Destacar, además, que no se han identificado pigmentos muy característicos y utilizados durante el siglo XVII, como, por ejemplo, los derivados de la industria del vidrio. ${ }^{27}$

24 GETTENS, R. J. , KÜHN, H. y CHASE, W. T. Lead white. En: Ashok ROY, ed. Artist's pigments: a handbook of their history and characteristics, vol. II. Washington: National Gallery and Archetype Publications, 1993, pp. 67-79.

25 WINTER, J. and WEST FITZHUGH, E. Pigments based on carbon. En: Barbara H. BERRI, ed. Artist's pigments: a handbook of their history and characteristics, vol. IV, Washington: National Gallery and Archetype Publications, 2007, pp. 1-37.

26 HELWIG, K. Iron oxide pigments: natural and synthetic. En: Barbara H. BERRI, ed. Artist's pigments: a handbook of their history and characteristics, vol. IV, Washington: National Gallery and Archetype Publications, 2007, pp. 39-109.

27 FERRER, P. y otros. New results in the characterization by Raman spectroscopy of yellow pigments used in ceramic artworks of the 16th and 17th centuries. Journal of Raman Spectroscopy, 2012, 43(11), pp. 1805-1810. 


\section{Procedencia y atribuciones diversas}

Desafortunadamente, la información documental sobre la historia de la versión de Barcelona es escasa, un aspecto que suele ser bastante frecuente en la investigación de muchas obras de arte. Hasta el momento, el primer dato relevante del que se tiene constancia se encuentra en un artículo que Adolfo Venturi publicó en el año 1900 y que dedicó a la pintura italiana que se conserva en el Museo de Bellas Artes de Budapest. En este artículo, Venturi no sólo hizo alusión a la versión que guarda el citado museo sino que expuso, además, las similitudes existentes con las versiones de Módena y Barcelona. Esta última, según afirma Venturi, pertenecía en aquel momento a la colección de Quincy Adams Shaw de Boston: "E copia dal Palma è pure il ritratto d'una donna bionda, simile agli altri della Galleria Estense in Modena e della raccolta di Mr. Quincy O [A] Schaw [Shaw] di Boston. Quest'ultimo sembra meglio modellato degli altri”. ${ }^{28}$

Aunque Venturi reprodujo la fotografía del retrato investigado, no se sabe si la llegó a ver directamente, ya fuese en Italia o en Boston. A pesar de ello, no dudó en afirmar que la versión de la colección de Quincy Adams (ahora en Barcelona) era la de mejor calidad. Por otra parte, y tal como se muestra en la cita anterior, consideró que las tres versiones tomaron como modelo una obra de Palma el Viejo. Esta hipótesis fue motivada quizás por una comparación con el retrato titulado La Bella (Fig. 6),

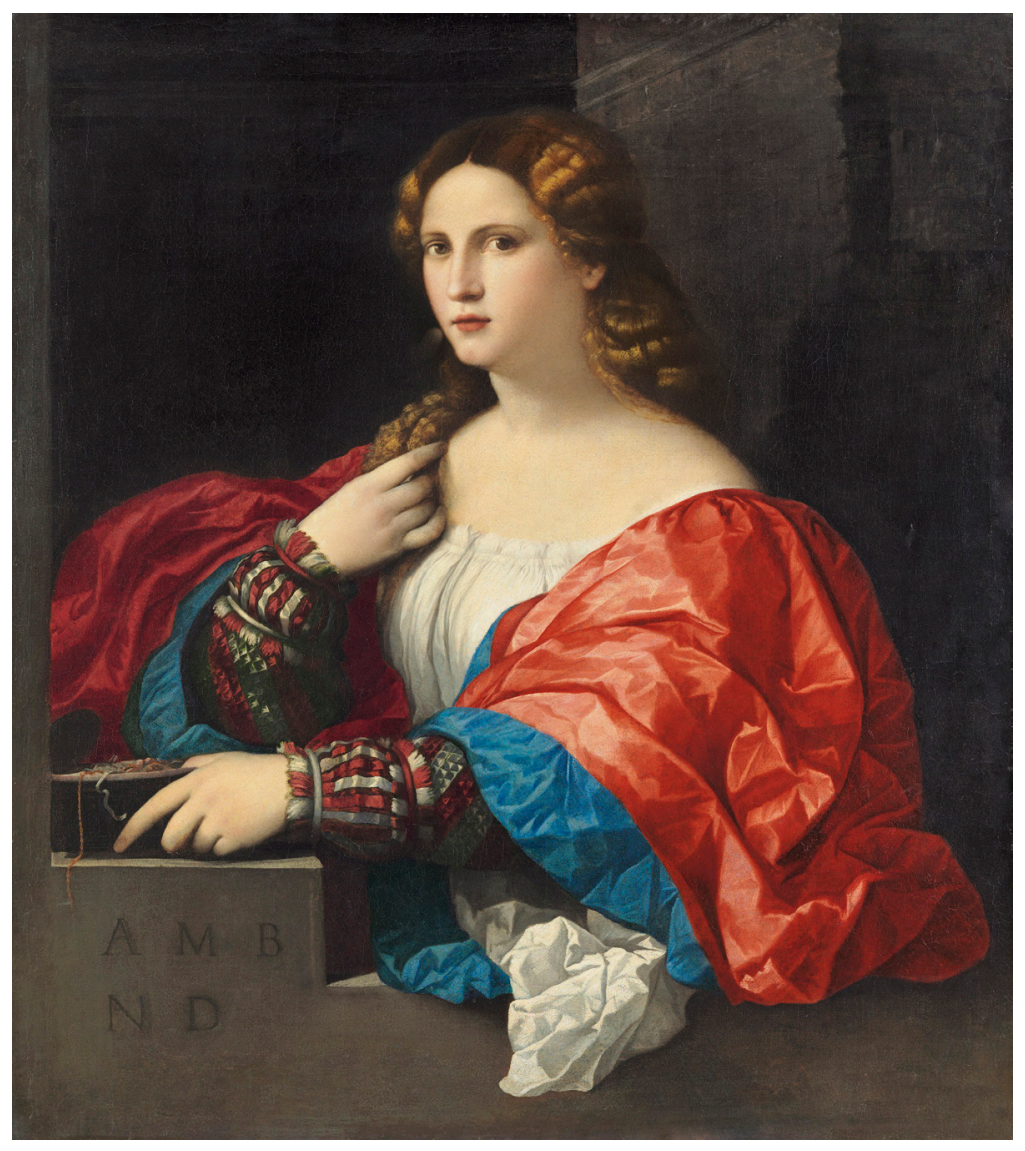

Fig. 6: Palma el Viejo. La Bella (I5I8-I520). Óleo sobre lienzo, 95 cm. x $80 \mathrm{~cm}$. Museo Thyssen Bornemisza. que representa también a una dama detrás de un parapeto en el cual hay varias letras inscritas. No obstante, los estilos de ambas obras difieren. Por ejemplo, el de La Bella, más aristocrático y distante, se aleja de la atmósfera íntima que caracteriza la versión de Barcelona. Además, en La Bella destaca una mirada directa hacia el espectador cargada de insinuación y voluptuosidad, mientras que la mirada de la joven en el retrato de Barcelona es de carácter más inocente y puro.

Por otro lado, la atribución que propuso Venturi también debió apoyarse en un grabado que Jan Van Troyen realizó siguiendo (según inscripción en dicho grabado) un modelo de Palma el Viejo y que representa a una dama con caracte-

28 VENTURI, A. I quadri di scuola italiana nella Galleria Nazionale di Budapest. L'Arte, rivista di storia dell'arte medievale e moderna. 1900, 3, pp. 216-217 y 233. [consulta: 10 de junio de 2020]. 
rísticas iconográficas más afines a las tres versiones que se presentan en este trabajo, aunque de estilos totalmente diferentes (Fig. 7). Ese grabado, junto con otros, se publicó en el conocido Theatrum Pictorium (1660) una colección que compiló David Teniers el Joven, por encargo del archiduque Leopoldo Guillaume de Austria, a partir de 243 obras que pertenecían a su colección. ${ }^{29}$

A pesar de que se desconoce la fecha en la que Quincy Adams pudo adquirir el retrato, sí sabemos que este importante coleccionista americano (Boston, 1825-1908) realizó varios viajes por Europa, sobre todo por Italia y Francia, donde parece que compró la mayor parte de las obras que constituían su colección. ${ }^{30}$ También se ha podido comprobar, gracias a la colaboración de Victoria Reed, conservadora del Museo de Bellas Artes de Boston, que la obra que nos ocupa estuvo depositada en préstamo en el citado

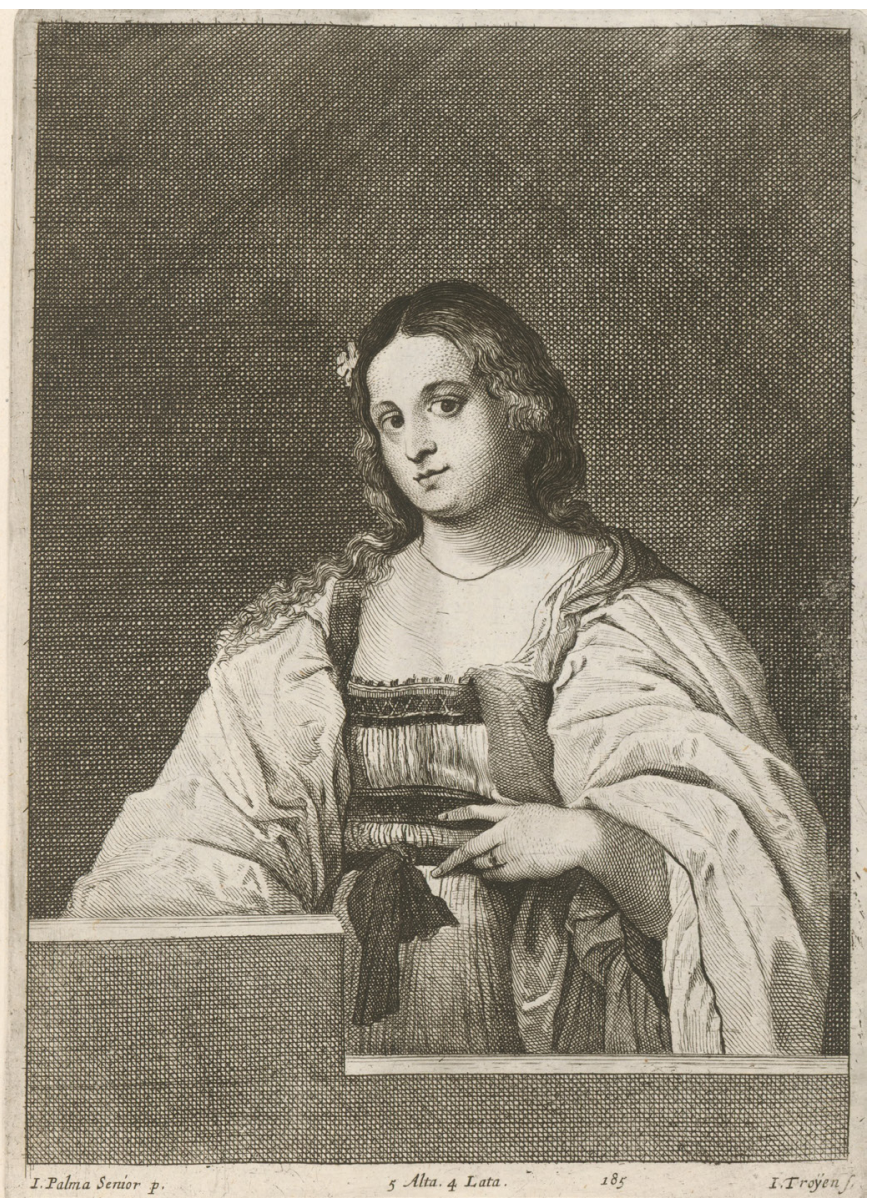

Fig. 7: Jan Van Troyen. Retrato de dama (1673). Slovak National Gallery. museo entre el 21 de mayo de 1913 y el 26 de marzo de 1917 con el título Retrato de Dama y catalogada como de escuela veneciana. Una vez cumplido el período de préstamo, el retrato se devolvió a los herederos de Quincy Adams, permaneciendo en manos de su hija Mariane hasta principios de los años cincuenta. En esa época, aunque en fecha desconocida, fue cuando Giovanni Castano (propietario de Castano Galleries de Boston) compró el retrato y, muy probablemente, hizo cambiar el bastidor viejo por el nuevo con extensores especiales (patentado por Charles Gordon Bearce en Massachusetts el año 1949). Con la intención de esclarecer la autoría de la obra, Castano encargó un estudio al historiador del arte Lionello Venturi (hijo de Adolfo Venturi) quien, en una carta fechada en octubre de 1954, expuso que el lienzo era "un excelente trabajo de la primera época de Tiziano"31. Según esta carta, Lionello Venturi había proporcionado al galerista americano un estudio pormenorizado del retrato, pero ese trabajo, desafortunadamente, no se ha podido localizar.

La atribución a Tiziano que realizó Lionello Venturi no sólo debió sustentarse en la presencia del

29 El Theatrum Pictorium, en su edición de 1673, está disponible en: https://archive.org/details/gri 33125009347911/page/n367/ mode/2up?q=palma+. [consulta: 12 de junio de 2020].

30 Ver parte de ella en: Museum of Fine Arts. Quincy Adams Shaw collection. Italian renaissance sculpture. Paintings and pastels by François Millet. Boston, 1918 [consulta: 10 de junio de 2020]. Disponible en: https://archive.org/details/quincyadamsshawc00muse/ page/n12/mode/2up.

31 "Rome, october 28, 1954 / Dear Mr. Castano, / I beg to confirm to you that the portrait of a young lady from de Quincy Adams Shaw collection is an excellent work of Titian of his early period. It compares with superiority to other replicas of the same subject. / I wrote for you the historical details about the picture some years ago. I can only confirm my great admiration for the picture, an example of the finesse and delicacy of the early period of Titian. / Yours sincerely, /LionelloVenturi." (Archivo particular, Barcelona). 


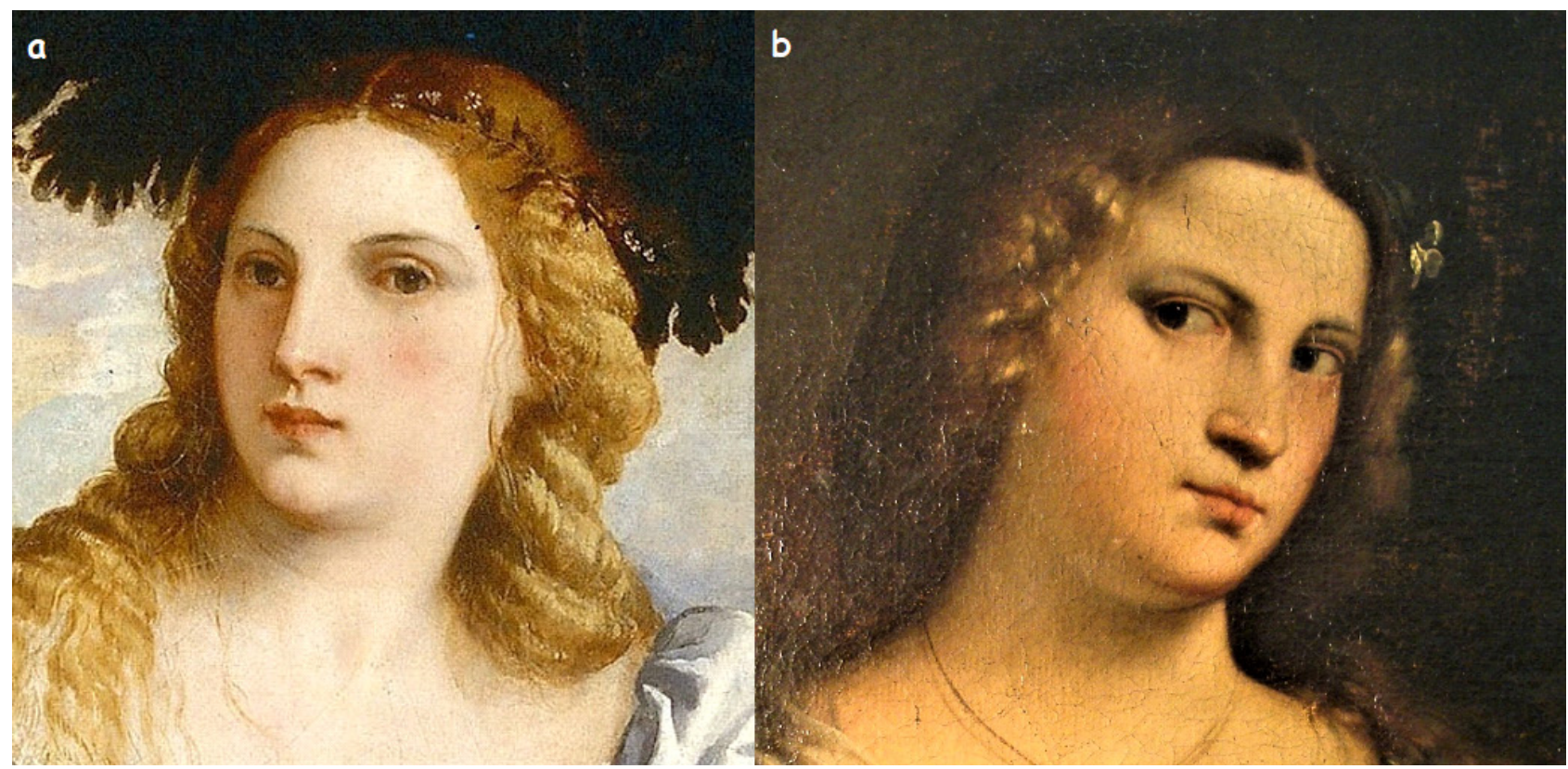

Fig. 8: Tiziano. Detalle del Amor sacro y amor profano (I5I4). Óleo sobre lienzo, II 8 cm. x 279 cm. Galería Borghese, Roma (a) comparado con el rostro de la retratada en la versión de Barcelona (b).

parapeto en las dos obras del maestro veneciano citadas ya anteriormente, sino también en el parecido con el prototipo de belleza ideal de la época, tal y como se puede comprobar si se compara el rostro que Tiziano ejecutó para su Amor sacro en 1514 con el rostro de la versión de Barcelona (Fig. 8).

Un tiempo después, a principios del año 1956, W. Gordon Wing, empresario de Texas, compró el retrato a Giovanni Castano. Este último, para legitimar la obra ante su cliente, le pidió un documento a la hija de Quincy Adams, Mariane Graeme Haugton, la cual accedió enviándole una carta fechada el 31 de enero de 1956 en la que relacionaba la obra con la escuela de Giorgione-Tiziano. ${ }^{32}$ En julio de ese mismo año, el historiador del arte Roberto Longui emitió, desde Florencia, una carta en la que opinó, igual que su colega Lionello Venturi, que la versión investigada era de mejor calidad que las versiones de Budapest y Módena, aunque, en cuanto al estilo, le parecía más apropiado relacionarla con la obra de Sebastiano del Piombo y no con la de Giorgione. ${ }^{33}$ Un año más tarde, en 1957, Bernard Berenson atribuyó la versión de Barcelona a Giovanni Cariani. ${ }^{34}$

En cuanto a la atribución a Sebastiano del Piombo, es evidente la afinidad que existe entre la obra investigada y el Retrato de joven como virgen sabia (Fig. 9). El parecido es apreciable en la delicadeza del dibujo de las manos y, sobre todo, en la posición de los dedos índice y corazón en forma de "V", un aspecto iconográfico que, como ya se ha comentado, une a las dos damas en el culto a Diana y las convierte en arquetipos femeninos como defensoras de la virtud y la castidad.

En relación a la atribución a Giorgione, no sólo es plausible por la atmósfera intimista que se respi-

32 "To whom it may concern: / I hereby declare that the Giorgione-Titian School Portrait, came from of my late father Quincy Adams Shaw and was part of his collection. My late father purchased the portrait in Europe. / Mariane Graeme Haugthon." (Archivo particular, Barcelona).

33 "Florence, july 6, 1956, / Thank you for the photos and your letter. From the reproduction your picture seems to me of a better quality than the paintings in Budapest and Modena [...] / But also your picture seems to me belong to the problem of Sebastiano del Piombo, not to the problem of Giorgione [...]". (Archivo particular, Barcelona).

34 BERENSON, B. Italian pictures of the renaissance. A list of the principal artists and their Works with an index of places. Venetian school. Vol. 1. Londres: The Phaidon Press, 1957, p. 54, lám. 732. 


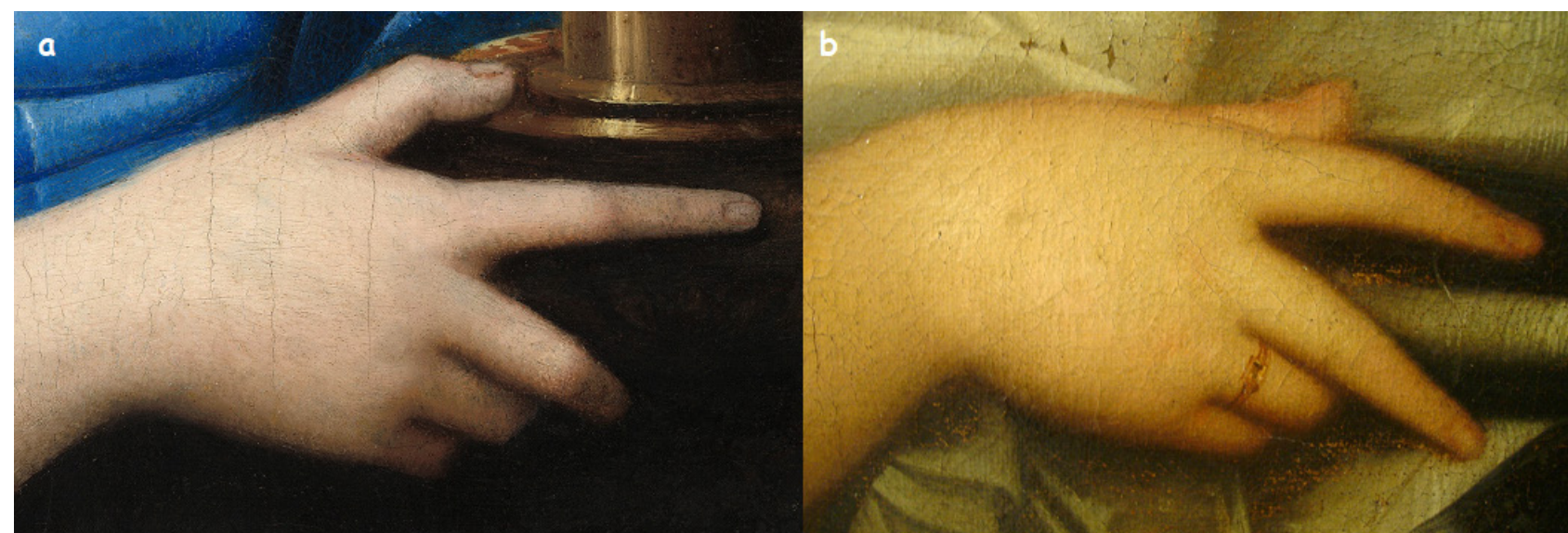

Fig. 9: Sebastiano del Piombo. Detalle de Retrato de joven como virgen sabia (I5I0). Óleo sobre lienzo, $54.7 \mathrm{~cm}$. x $47.5 \mathrm{~cm}$. Galería Nacional de Arte, Washington (a) comparado con la mano derecha en la versión de Barcelona (b).

ra en toda la obra, sino también por algunos detalles concretos como, por ejemplo, en el dibujo de los labios y las comisuras de la retratada que evocan la delicadeza del estilo de Giorgione en el Joven con flecha (Fig. 10). De hecho, Claire Garas, en 1964, comentó que la versión conservada en Budapest tenía un aire "muy giorgionesco", igual que las otras dos versiones conocidas (Módena y Barcelona), así como también otro retrato conservado en el Museo de Budapest (catalogado como de pintor veneciano) que muestra, sin lugar a dudas, a la misma modelo representada unos años después (Fig. 11). ${ }^{35}$ El vestido negro y el pelo recogido son elementos que aluden a su nueva condición de mujer casada, aunque vuelve a posar con el gesto de "V" invertida, señal que seguía vinculada a la defensa de los conceptos de virtud y castidad. Destacar que Garas, en cuanto a la versión de Barcelona, la ubicaba entonces en el Museo de Bellas Artes de Houston. Y parece ser que allí permaneció durante varios años, ya que Rodolfo Pallucchini también la localizó en el mismo museo cuando aludió a ella en 1983 como una obra de Giovanni Cariani. ${ }^{36}$

A partir de entonces, parece que la pista sobre la localización del retrato se perdió en los círcu-

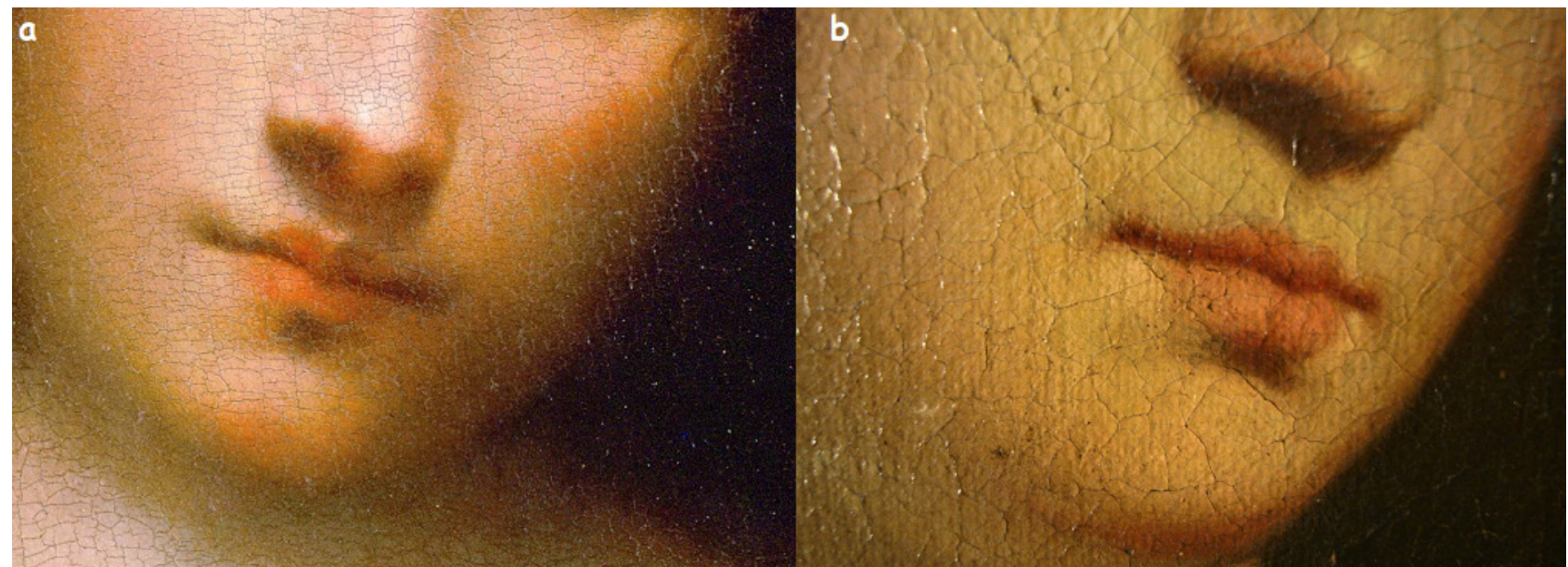

Fig. I0: Giorgione. Detalle de Joven con flecha (I505). Óleo sobre tabla, $48 \mathrm{~cm}$. x $42 \mathrm{~cm}$. Museo de Bellas Artes, Viena (a) comparado con un detalle de los labios en la versión de Barcelona (b).

35 GARAS, C. Giorgione et giorgionisme au XVIIè siècle. Bulletin du Musée Hongrois des Beaux Arts. 1964, 25, pp. $73-76$ [consulta: 12 de junio de 2020]. Disponible en: https://library.hungaricana.hu/en/view/ORSZ_SZEP_Kozl_025/?pg=52\&layout=S

36 PALLUCCHINI, R. y ROSSI, F. Giovanni Cariani. Milán: Silvana, 1983, pp. 299-300. 
los científicos, aunque no por ello dejó de estar presente en la historiografía, sobre todo como información adicional a trabajos dedicados a las versiones de Budapest y Módena, obras que también han tenido atribuciones muy diversas a lo largo de la historia, incluyendo a artistas como Giorgione, Tiziano, Sebastiano del Piombo y Palma el Viejo. ${ }^{37}$ No obstante, parece que se ha llegado a un consenso en atribuir ambas versiones a Giovanni Cariani, el estilo del cual se forjó a partir de una actitud muy receptiva a lo que ocurría en pintura durante la primera década del siglo XVI en Venecia. Su obra destaca, entre otros aspectos, por un tratamiento suave de la luz, el uso de una paleta neutra y sobria, por la naturalidad de la pose de los modelos y la suave expresión de sus rostros. Pero lo más importante es que las obras de

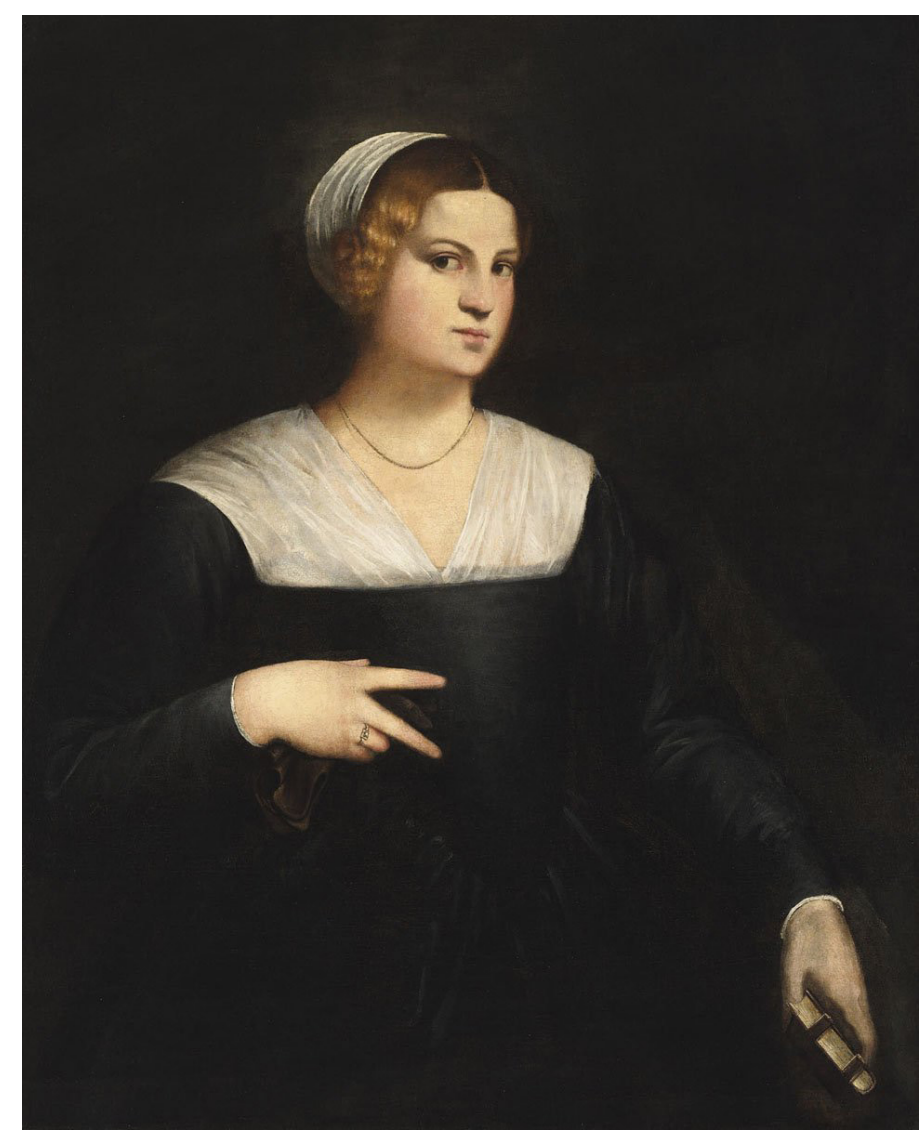

Fig. I I: Pintor veneciano (anónimo). Retrato de dama (c. I540). Óleo sobre lienzo, $93.5 \mathrm{~cm}$. x $76 \mathrm{~cm}$. Museo de Bellas Artes, Budapest.

su primera época evocan un aire tan giorgionesco que algunos autores atribuyeron a Giorgione obras que actualmente se aceptan como de Cariani. ${ }^{38}$ Simone Fracchinetti lo justifica diciendo que Cariani pudo haber conocido a Giorgione a través del coleccionista y mecenas Gabriele Vendramin y, lógicamente, a partir de ahí y de la asimilación directa de su obra, se empapó del estilo del maestro, así como del de otros pintores contemporáneos, como, por ejemplo, Sebastiano del Piombo. ${ }^{39}$

\section{Consideraciones finales y conclusiones}

Hay que resaltar, en primer lugar, que todos los artistas citados en este trabajo (Giorgione, Tiziano, Sebastiano del Piombo, Palma el Viejo y Giovanni Cariani), ${ }^{40}$ ejercieron su actividad en la misma ciudad (Venecia) durante la primera mitad del siglo XVI y, por lo tanto, se influyeron entre ellos tanto en estilo como en la aplicación de diferentes tipos de composiciones como, por ejemplo, en el uso del

37 GIORDANI, N. y SCALINI, M. Rinascimento privato. Aspetti inconsueti del collezionismo degli Este da Dosso Dossi a Brueghel. Milán: Silvana, 2010, pp. 150-151.

38 FACCHINETTI, S. Gli esordi di Giovanni Cariani. Bérgamo: Press R3, 2016, p. 7-9 [consulta: 12 de junio de 2020]. Disponible en: ttps://www. academia. edu/31708003/Gli_esordi_di_Giovanni_Cariani_Bergamo_2016

39 FACCHINETTI, S. y GALANSINO, A. In the age of Giorgione. Royal Academy of Arts, Londres, 2016, p. 39 y 146-147 [consulta: 12 de junio de 2020]. Disponible en: https://www.academia.edu/27975357/In the Age_of_Giorgione exhibition_catalogue edited_by_S_Facchinetti_and_A_Galansino_Royal_Academy_of_Arts_London_2016.

40 Excepto Sebastiano del Piombo que se trasladó a Roma en 1511. 
parapeto con letras inscritas. Por ello es normal que hayan existido diversas opiniones en cuanto a la posible autoría del retrato motivo del presente trabajo. Destacar, además, que esos artistas realizaron sus retratos con parapeto durante las dos primeras décadas del siglo XVI, siendo el más antiguo el Retrato Giustiniani de Giorgione (c. 1505) y el más moderno La Bella de Palma el Viejo (c. 1518-1520). Por eso, es más que probable que, por estilo y composición, el retrato de Barcelona se realizara en esa época o, como máximo, durante la primera mitad del siglo XVI. Esta conclusión es consistente, además, con la época de uso de los pigmentos identificados mediante el análisis molecular no destructivo realizado con espectroscopia Raman. Tal y como se ha comentado, la obra se ejecutó con una paleta muy sobria compuesta fundamentalmente de blanco de plomo, tierras amarillas, ocres y rojas (hematites) a base de hidróxidos y óxidos férricos y carbón de origen vegetal, todos ellos considerados pigmentos históricos. Resaltar, además, que tan importante ha sido identificar estos pigmentos como no identificar otros descubiertos y bien datados en siglos posteriores como, por ejemplo, pigmentos amarillos a base de plomo, estaño y/o antimonio, muy relacionados con la industria del vidrio en el siglo XVII.

Por otra parte, ha quedado argumentado que la letra "V" del parapeto seguramente simboliza el lema latino Virtus omnia vincit, un tópico literario que fue ampliamente utilizado en los círculos humanísticos de las cortes principescas italianas. Por eso, es lógico que los artistas más importantes de principios del siglo XVI, imbuidos de la ideología humanista, quisieran reflejar en sus obras y, sobre todo, en los retratos, conceptos sublimes como, en este caso, la Virtud. Pero también existe una lectura iconológica más arcana y hermética que tiene que ver con la posición de los dedos de la modelo en forma de "V" invertida, un gesto que simbolizaría un culto pagano a la diosa Diana basado en la defensa de los conceptos de castidad y virginidad. En la obra investigada destaca el naturalismo que emana, de manera sencilla, de cada uno de los elementos que la integran, y se puede apreciar cómo el artista quiso ir más allá de la mera representación física para captar el alma de la modelo evocando un arquetipo de belleza casta y de virtud femenina ideal, a la manera preconizada por Leonardo da Vinci.

La observación e interpretación de múltiples micro y macrofotografías de la obra obtenidas con binocular estereoscópica han sido esenciales para apreciar de manera objetiva ese naturalismo en los detalles y la calidad de la técnica en las veladuras. Asimismo, los resultados del estudio con reflectometría I. R. , que muestran un dibujo preliminar realizado al carbón, han afianzado la opinión de que el artista era un pintor técnicamente muy dotado.

Finalmente, con independencia de las atribuciones existentes, los resultados obtenidos en la presente investigación, tanto científicos como histórico-artísticos y documentales, nos llevan a sugerir, como una nueva propuesta razonable, la hipótesis de que la versión de Barcelona pudo ser pintada por Giovanni Cariani y que, a su vez, esa versión debió servir de modelo para las versiones de Módena y Budapest. 


\section{Bibliografía}

ALBERTI, Leon Battista. Tratado de pintura. México D. F. : Universidad Autónoma Metropolitana, 1988. ISBN $970-$ 654-317-1.

BEARCE, Charles Gordon. Frame for artists' canvases with expanders. US2491600A, United States Office, 1949 [consulta: 9 de junio de 2020]. Disponible en: https://patents.google.com/patent/US2491600A/en.

BELL, Ian. M. CLARK, Robin. J. H. y GIBBS, Peter. J. Raman spectroscopy library of natural and synthetic pigments (pre-1850). Spectro chimica Acta. Part A. 1997, vol. 53, pp. 2159-2179. ISSN 1386-1425.

BERENSON, Bernard. Italian pictures of the renaissance. A list of the principal artists and their works with an index of places. Venetian school. Vol. 1. Londres: The Phaidon Press, 1957. ISBN 0714814865.

BONIFACIO, Giovanni. L’Arte de Cenni. Vicenza, 1616 [consulta: 9 de junio de 2020]. Disponible en: https://archive. org/details/lartedecenniconl02boni/page/n3/mode/2up.

CALVESI, Maurizio. La morte di bacio. Saggio sull'ermetismo di Giorgione. Storia dell'Arte. 1970, nº 7-8, pp. 179-233. ISSN 0392-4513.

CHILLÓN, M. Concepción, FERRER, Perla, RUIZ-MORENO, Sergio y LÓPEZ GIL, Alejandro. Avoiding frauds: Raman spectroscopy, a fundamental tool in the analysis of artworks. En: Rogério AMOÊDA, Sérgio LIRA y Cristina PINHEIRO, eds. Heritage 2012, 3rd International Conference on Heritage and Sustainable Development, vol. 2. Portugal: Green Lines Institute, 2012, pp. 805-814. ISBN 978-989-95671-5-3.

FACCHINETTI, Simone. y GALANSINO, Arturo. In the age of Giorgione. Royal Academy of Art. Londres, 2016. [consulta: 12 de junio de 2020]. Disponible en: https://www.academia.edu/27975357/In the Age of Giorgione exhibition_catalogue_edited_by_S_Facchinetti_and_A_Galansino_Royal_Academy_of_Arts_London_2016. ISBN 978-1-910350-26-3.

FACCHINETTI, Simone. Gliesordi di Giovanni Cariani. Bérgamo: Press R3, 2016 [consulta: 12 de junio de 2020]. Disponible en: https:/www.academia.edu/31708003/Gli_esordi_di_Giovanni_Cariani_Bergamo_2016. ISBN 9788894162172.

FERRER, Perla y otros. New results in the characterization by Raman spectroscopy of yellow pigments used in ceramic artworks of the 16th and 17th centuries. Journal of Raman Spectroscopy, 2012, 43 (11), pp. 1805-1810. ISSN 1097-4555.

GARAS, Claire. Giorgione et giorgionisme au XVIIè siecle. Bulletin du Musée Hongrois des Beaux-Arts, 1964, 25, pp. 51-80 [consulta: 12 de junio de 2020]. Disponible en: https://library.hungaricana.hu/en/view/ORSZ_SZEP

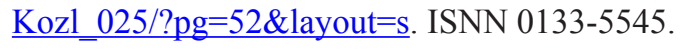

GAYO, Ma Dolores y JOVER, Maite. Evolución de las preparaciones en la pintura de los siglos XVI y XVII en España. Boletín del Museo del Prado, 2010, vol. 28, n 46, Madrid, pp. 39-59. ISSN 0210-8143. 
GETTENS, Rutherford, J. , KÜHN, Hermann y CHASE, W. T. Lead white. En: Ashok ROY, ed. Artist's pigments: a handbook of their history and characteristics, vol. II. Washington: National Gallery and Archetype Publications, 1993, pp. 67-79. ISBN 978-1-904982-75-3.

GIORDANI, Nicoletta y SCALINI, Mario. Rinascimento privato. Aspetti inconsueti del collezionismo degli Este da Dosso Dossi a Brueghel. Milán: Silvana, 2010. ISBN 978-8836617135.

HELWIG, Kate. Iron oxide pigments: natural and synthetic. En: Barbara H. BERRI, ed. Artist's pigments: a handbook of their history and characteristics, vol. IV, Washington: National Gallery and Archetype Publications, 2007, pp. 39-109. ISBN 978-1-904982-23-4.

Legge 1 Giugno 1939, nº 1089. Tutela della cose d'interesse artistico o storico, en: Gazzeta Uffiziale della Repubblica italiana, $\mathrm{n}^{\circ}$ 184, 8 de agosto de 1939 [consulta: 9 de junio de 2020]. Disponible en: https://librari.beniculturali.it/export/ $\underline{\text { sites/dgbid/it/documenti/Normativa/Legge_1_giugno_1939_n_1089.pdf. }}$

MARANGON, Giorgia. Las cortesanas venecianas y la moda. En: Mujer, cultura y comunicación: realidades e imaginarios. IX Simposio Internacional de la Asociación Andaluza de Semiótica. Sevilla: Alfar, 2001, pp. 518-522 [consulta: 8 de junio de 2020]. ISBN 84-89673-84-5. Disponible en: https://idus.us.es/bitstream/handle/11441/60783/ MARANGON_Giorgia.\%20pdf?sequence=1\&isAllowed $=\mathrm{y}$.

MÁRQUEZ, Jaime y otros. La moda femenina en Italia del siglo XVI al siglo XVIII. En: La moda femenina en la Edad Moderna. Universidad de Cádiz, 2013. [consulta: 8 de junio de 2020]. Disponible en: https://www.academia.edu/5111954/ LA_MODA_FEMENINA_EN_LA_EDAD_MODERNA.

MORE, Nicolás. Résumé de la restauration du tableau "Violante" de Giovanni Cariani. Bulletin du Musée Hongrois des Beaux-Arts. 1979, 52, pp. 89-96 [consulta: 9 de junio de 2020]. Disponible en:

https://library.hungaricana.hu/en/view/ORSZ SZEP Kozl 053/?query=More\%2C\%20Nicolas\&pg=90\&layout=s. ISSN 0133-5545.

MUSEUM OF FINE ARTS. Quincy Adams Shaw collection. Italian renaissance sculpture. Paintings and pastels by François Millet. Boston, 1918 [consulta: 10 de junio de 2020]. Disponible en: https://archive.org/details/ quincyadamsshawc00muse/page/n12/mode/2up.

PALLUCCHINI, Rodolfo y ROSSI, Francesco. Giovanni Cariani. Milán: Silvana, 1983. ISBN 8836600379.

SYSON, Luke. Testimonio de rostros, recuerdo de almas. En: Miguel FALOMIR ed. El retrato del Renacimiento. Madrid: El Viso, 2008, pp. 23-39. ISBN 978-84-95241-56-6.

THOMPSON, Nancy. VV and related inscriptions in Giorgione, Titian and Dürer. The Art Bulletin. 1975, n 57, pp. 346356. [consulta: 7 de junio de 2020]. Disponible en: https://www.jstor.org/stable/3049402?refreqid=excelsior\%3A25a22f b7404e06a969ec59c9630b0ad2\&seq=1. ISSN 00043079.

VENTURI, Adolfo. I quadri di scuola italiana nella Galleria Nazionale di Budapest. L'Arte, rivista di storia dell'arte medievale e moderna. 1900, nº 3, pp. 216-217 y 233. [consulta: 10 de junio de 2020]. ISSN 2365-6700. 
WINTER, John and WEST FITZHUGH, Elisabeth. Pigments based on carbon. En: Barbara H. BERRI, ed. Artist's pigments: a handbook of their history and characteristics, vol. IV, Washington: National Gallery and Archetype Publications, 2007, pp. 1-37. ISBN 978-1-904982-23-4.

ZANCHI, Mauro. Sotto il segno di Diana. Il gesto a V rovesciata nell'arte del Cinquecento: letture iconologiche. Art e dossier, 2014, no 315, pp. 72-77. ISSN 0394-0179. 